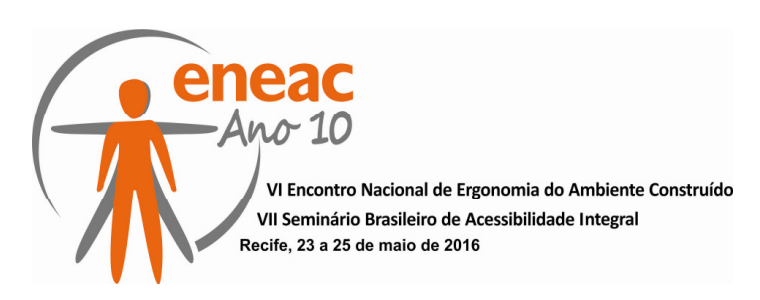

\title{
COMO SALVAR VIDAS DE PESSOAS COM DEFICIÊNCIAS EM EMERGÊNCIAS? A INVENÇÃO E O DESENVOLVIMENTO DE NOVOS PISOS TÁTEIS FOTOLUMINESCENTES
}

\author{
ZURBA, Nadia Khaled \\ Universidade Federal do Ceará, Professora Doutora \\ Centro de Tecnologia, Av. da Universidade 2890, 60.020-181, Fortaleza, CE \\ E-mail: $\underline{\text { 2698@ua.pt }}$
}

\section{INTRODUÇÃO}

«Accessibilidade» para todos é um direito fundamental numa sociedade inclusiva considerando as condições especiais para a mobilidade, participação e integração nas actividades da vida. A «deficiência» não é termo absoluto no mundo e muitos países não possuem estatísticas exatas do número de pessoas que vivem com deficiências funcionais. A exemplo da União Européia e alguns Estados Membros, do ponto de vista econômico (e.g. elegibilidade a benefícios), social (e.g. inclusão em atividades), e médico (e.g. diagnóstico de doenças), existem grandes discrepâncias entre os países da Europa que adoptam o conceito de pessoas com deficiências [1]. No âmbito internacional, zelar pelos direitos dessas pessoas é uma prioridade da Organização das Nações Unidas para a Educação, Ciência e Cultura (UNESCO) dedicada à uma estimativa dos mais de 330 milhões portadores de deficiências que vivem no mundo todo [2].

Em sinergia e em continuidade às ações realizadas em prol da igualdade de oportunidade para todos, arquitetos, engenheiros e outros profissionais que participam na concepção e construção do espaço físico devem promover a construção adequada de medidas inovadoras destinadas a garantir a acessibilidade integral de pessoas com deficiências, como processo de realização da igualdade de oportunidades em todas as esferas da sociedade. Neste trabalho, "pessoas com deficiências» enquadra-se como um termo que define um grupo não-homogêneo de indivíduos de uma comunidade com uma pluralidade de condições funcionais de «necessidades humanas especiais», sejam a partir de deficiências físicas, temporárias ou permanentes, incluindo deficiência motora, sensorial, cognitiva e respiratória - também incluindo utilizadores com baixa mobilidade, baixa visão ou dependência de tecnologias assistivas, tais como cadeira de rodas e pavimentos táteis. ${ }^{[3-}$

11] As necessidades especiais destas pessoas precisam ser atendidas, supridas com tecnologias efectivas que auxiliem na sua locomoção, evitando assim a exclusão social.

\section{ESTADO DA ARTE}

Pisos cerâmicos para acessibilidade, em porcelanato tátil, foram desenvolvidos no Brasil, cuja tecnologia [9] foi premiada pela Sociedade Brasileira de Cerâmica, em 2004, e finalista do $18^{\circ}$ Prêmio Design Museu da Casa Brasileira, categoria de Novas Ideias/Conceitos. A inovação tecnológica de tais pisos cerâmicos para acessibilidade foi implementada em 2007 [9-11], e produtos similares também foram produzidos em Portugal. No entanto, apenas placas táteis foram criadas, sem que alguma solução fosse apresentada com a propriedade de fotoluminescência para sinalização em caso de emergência. 


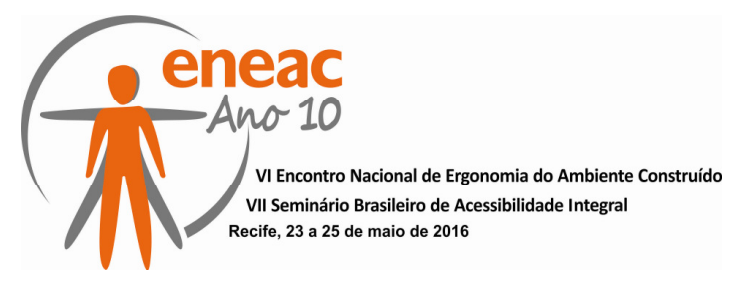

Em paralelo, para aplicações em sinalizações, materiais avançados com «fotoluminescência persistente (FL)» têm sido desenvolvidos e implementados em vários países da Europa, regulamentados pela rigorosa norma alemã DIN 67510 [12]. Nos Estados Unidos, um sistema FL muito eficaz vem sendo usado nas rotas de evacuação e saídas de emergência da Estação Espacial Internacional (do inglês, "International Space Station ISS") da NASA [13], utilizando o material luminescente "aluminato de estrôncio» na fabricação de placas de sinalização, referência mundial de boa emissão luminosa, mas cujo sistema não é adaptado a pessoas com deficiências.

Uma padronização internacional sobre estes materiais e produtos fotoluminescentes para evacuação de emergência é particularmente descrita pela Associação Britânica de Produtos de Segurança Fotoluminescentes (sigla em inglês, PSPA) que estabelece alguns critérios e recomendações para a instalação de materiais e produtos FL [14]. Entretanto, tanto a DIN 67510 como o código de práticas da PSPA deixam uma séria lacuna no que se refere a sistemas FL para pessoas com deficiências. A PSPA propõe que soluções de sistemas FL de evacuação horizontal para pessoas com necessidades especiais sejam tratadas por especialistas (Art. 3.2 - Special Needs) [14], comprovando a falta de soluções específicas para a acessibilidade das pessoas com deficiências, em emergências.

\section{METODOLOGIA}

A metodologia utilizada no desenvolvimento da tese teve como fundamentação teórica e prática a experiência adquirida em atividades realizadas, entre os anos 1998 e 2006, no desenvolvimento de novos produtos/brinquedos para a reabilitação de pessoas com deficiências, em eventos organizados pela instituição Fördern durch Spielmittel e.V., Berlin, no âmbito da Comissão da UNESCO da Alemanha, Bonn [15-16]. O contato estreito e interativo de aprendizado efetuado junto aos portadores de deficiências internacionais foi útil também para revelar necessidades multiculturais que tais utilizadores requerem no processo de reabilitação, bem como as diferentes políticas e estratégias que suportam os direitos e qualidade de vida de tais cidadãos. Na área experimental de materiais, novos nanomateriais luminescentes foram preparados pelo método de síntese via estado sólido [17]. O trabalho teórico-prático compreendeu o conteúdo da Tese de Doutorado em Ciência e Engenharia de Materiais, com transversalidade nas áreas da Arquitetura, Design, Física e Medicina [17].

\section{RESULTADOS E DISCUSSÃO}

\section{Como salvar vidas de pessoas com deficiências em emergências?}

No caso de situações de emergência, o «direito de ir e vir» dos portadores de deficiências é imprescindível como «fator vital» que assegure a possibilidade de evacuação em tempo hábil, com integridade física e segurança. Entretanto, ocorre um sério problema de segurança pública até que se desenvolvam meios suficientes de compensar as restrições de mobilidade de pessoas com deficiências no processo de evacuação emergências. Em geral, essas pessoas dispõem de menores velocidade de locomoção, resistência física e agilidade e, por isso, a locomoção ocorre com menor autonomia. A adequação do espaço físico para a evacuação de áreas de risco, incluindo edifícios, hospitais, centros de reabilitação e uso residencial e institucional, constitui-se, assim, num fator prioritário de inclusão social para salvar vidas de pessoas portadoras de deficiências em emergências.

A apresentação no «VI Encontro Nacional de Ergonomia do Ambiente Construído e VII Seminário Brasileiro de Acessibilidade Integral - ENEAC 2016» traz à luz uma resposta de pesquisa, com os resultados de um estudo científico multidisciplinar que demonstra novas soluções para modificar a realidade das pessoas com deficiências, em emergências. 


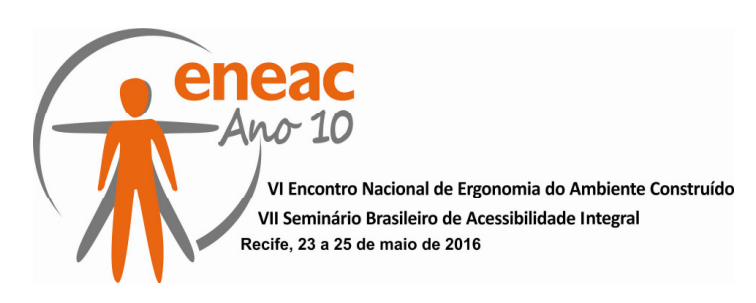

Tal resposta consiste no aproveitamento da propriedade de luminescência persistente (emissão de luz por mais de 10 horas) de novos nanomateriais (uma dimensão menor que $100 \mathrm{~nm}$ ) aplicados em um novo produto inventado com esta propriedade para a acessibilidade [17]. Mais especificamente, a investigação resultou na aplicação da propriedade da luminescência persistente de $\mathrm{SrAl}_{2} \mathrm{O}_{4}: \mathrm{Ce}$ (III), Dy, Eu em sistemas de acessibilidade de emergência, incluindo utilizadores portadores de deficiências.

O trabalho revela a importância máxima de salvar vidas de pessoas com deficiências em emergências, através da invenção, ao nível mundial, de novos dos «Pisos Cerâmicos Táteis Fotoluminescentes» [17]. Além disso, culminou-se na proposição de medidas de prevenção das deficiências evitáveis in vivo através da invenção de um novo «equipamento biomédico oftalmológico para testar células retinais fotorreceptoras», seja por proporcionar uma maior segurança aos seus utilizadores nos percursos de pedestres (pisos), ou pelo diagnóstico precoce de doenças oftalmológicas (equipamento), para além de promover um processo contínuo de reabilitação concomitante à utilização de tais tecnologias assistivas. Da generalidade dos diversos tipos de deficiências, maior detalhe foi atribuído ao estudo das deficiências visuais, na prevenção de doenças oftalmológicas e na visibilidade de materiais fotoluminescentes.

No campo da nanociência, o material «aluminato de estrôncio co-dopado com cério (III), disprósio (III) e európio (II), $\mathrm{SrAl}_{2} \mathrm{O}_{4}: \mathrm{Ce}^{3+}, \mathrm{Dy}^{3+}, \mathrm{Eu}^{2+} »$, revelou-se extraordinariamente avançado a partir da sua nova nanoestrutura (nanotubos luminescentes), e com novas propriedades multifuncionais (processo de encapsulamento), elevando a contingência da fotoluminescência persistente às pessoas com deficiências.

Tais resultados foram publicados em 6 Patentes de Invenção abordadas nos tópicos desta apresentação, concedidas pelo Instituto Nacional da Propriedade Industrial (INPI) entre os anos de 2007 e 2012. O caráter inovador desse trabalho intitulado "NANO-EMERGÊNCIA» foi galardoado com o «Prémio Maria Cândida da Cunha 2009, do Instituto Nacional para a Reabilitação - INR», e também publicado como livro, na Alemanha. O conjunto de soluções desenvolvidas para a inclusão das pessoas com deficiência nas rotas de evacuação integram o presente objeto de discussão no «ENEAC 2016». Refira-se, por fim, que em continuidade à criação da invenção, os novos «Pisos Táteis Fotoluminescentes» estão em atual estágio de desenvolvimento, com o auxílio do Conselho Nacional para o Desenvolvimento Científico e Tecnológico - CNPq.

\section{CONCLUSÕES}

A tese transversal teve por objetivo desenvolver uma solução para resolver um grave problema de segurança pública e da inclusão social das pessoas com deficiência em situações de emergência, através da prospecção da propriedade de luminescência persistente em rotas de evacuação. Alguns problemas derivados foram abordados na área específica de Ciência dos Materiais e Engenharia: (1) desenvolver nanomateriais luminescentes multifuncionais; (2) alcançar os requisitos de emissão de luz no escuro para sinalização de emergência; (3) identificar potenciais aplicações in vivo. A solução integrada dos novos nanotubos, nanoarames e cápsulas luminescentes, e a visão cromática personalizada no fundo preto, testada em um novo equipamento oftalmológico, mesmo por um grupo estatístico crítico de pessoas com retinopatia diabética, suportam a aplicação dos pisos cerâmicos fotoluminescentes para áreas de risco e emergência, cuja facilidade é acessível para todas as pessoas. 


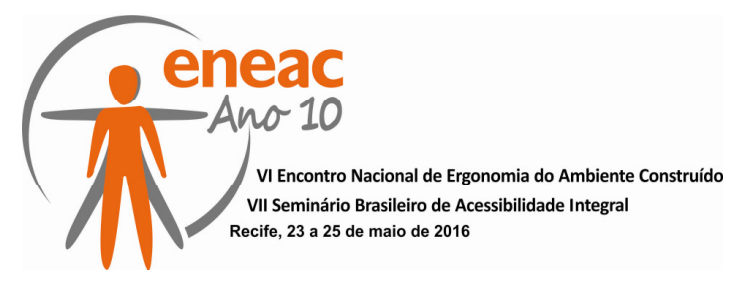

\section{REFERÊNCIAS}

[1]. EUROSTAT - European Commission Statistical Committees, Population and Social Conditions, 2009, Luxembourg; 2010: A Europe Accessible for All. Report from the Group of Experts set up by the European Commission, 2003, 27p. In: http://www.polisubd.net/UserFiles/File/EU_Access2010_Report-1.pdf.

[2]. UNESCO - United Nations for Education, Science and Culture Organization, UN agency citing WHO - World Health Organization: people with disabilities, UNESCO Program Bildung for Kinder in Not, 2006.

[3]. ANSI - American National Standard Institute, ANSI ICC/A117.2-1998 Accessible and Usable Buildings and Facilities, Falls Church, VA: International Code Council. 2003.

[4]. ADAAG - Americans with Disabilities Act Accessibility Guidelines for Buildings and Facilities, ADAAG 4.29, 1991. In: http://www.access-board.gov/adaag.

[5]. ISO - International Organization for Standardization, ISO/TC 173, Assistive products for persons with disability. 1999.

[6]. J. Tavares, E. Martins, M. Dischinger, N. K. Zurba, Pilot Project of Technical Solutions for Urban Accessibility of Handicapped People and Elaboration of Municipal Law Facilities $\mathrm{N}^{\circ}$. 2.153.784, Institute of Urban Planning of Florianópolis, IPUF, 1998.

[7]. M. Dischinger, N. K. Zurba, Accessibility in Historical Centre of Florianopolis. Technical Report of the Institute of Urban Planning of Florianopolis. IPUF. 1998.

[8]. N. K. Zurba, M. Dischinger, Accessibility in Public Urban Spaces. IV Proc. ENEPEA, 1998, 1, 33-4.

[9]. N. K. Zurba, Ceramic tiles with warning devices for people with disabilities, Appl. Patent, 2003, BRMU83026479, BRMU84004142.

[10]. N. K. Zurba, Methodology of the Design Process and Classification of Floor Tiles for Accessibility Applied in Porcelain Stoneware Material, Master Dissertation Mat. Sc. Eng. 2003, Federal University of Santa Catarina, 106p.

[11]. Eliane Ceramic Tiles, Arqtec, 2007.

[12]. DIN - Deutsches Institut für Normung e.V., DIN 67510. German Industrial Standard: Photoluminescent pigments and products; escape route system, 2004.

[13]. NASA - National Aeronautics and Space Administration. NASA's Space Operations Mission Directorate - Photoluminescent System for International Space Station (ISS), Scientific and Technical Information, 2004, Washington DC, US.

[14]. PSPA - Photoluminescent Safety Products Association. British Association of Normalization of Photoluminescent Products and Materials: a) PSPA Standard 002, Art. 3.2. Special Needs, 1997; b) PL Standard: Emergency Wayfinding Guidance Systems, Guide to the Use of Photoluminescent Safety Markings, 2008; c) Guide to the Use of Photoluminescent Safety Markings, Part 1: Egress Markings in Stairwells, 2008. In: http://www.pspa.org.uk.

[15]. Recentes desenvolvimentos para a reabilitação de pessoas com deficências, veja: N. K. Zurba in UNESCO Symposium, Workshop and Exhibition Toys for Rehabilitation: a) Humanism by (Bio)Materials - An Introduction for Accessibility and Rehabilitation. Deutschen UNESCO-Kommission, Bielefeld, Deutschland, 2006; b) Universal Design in Toys". UNESCO Toys for Children's Rehabilitation, Symposium presentation. Chinese and Deutschen UNESCO-Kommission, Anji, China, 2003; c) Designing for Handicapped People, UNAM and Deutschen UNESCO-Kommission, Mexico City, Mexico, 1998.

[16]. Experiência em centro de reabilitação, veja: Zurba N. K. in UNESCO Spielkulturen, A. Heinzelmann, C. Sonntag, R. Elbracht. Deutschen UNESCO-Kommission, Wissen Media Verlag, 2007, 1, 12. ISBN 978-3-577-10200-1. In: http://www.unesco.de.

[17]. Zurba N.K., Ferreira J.M.F, Nano-emergency: Persistent luminescence of $\mathrm{SrAl}_{2} \mathrm{O}_{4} \mathrm{Ce}(\mathrm{III})$, Dy, Eu nanotubes, nanowires and core-shells for people with disabilities. Saarbrücken: LAP LAMBERT Academic Publishing AV Akademikerverlag GmbH \& Co. KG, Germany, ISBN 978-3-659-33404-7, 2013, 204p. 